\title{
GW23-e2584 A PROSPECTIVE SINGLE-CENTRE STUDY OF FUNGAL INFECTIVE ENDOCARDITIS
}

doi:10.1136/heartjnl-2012-302920s.5

Sun Xiaolu, Wang Guogan, Zhang Jian, Sun Xiaolu. Fuwai Hospital

Objectives Fungal infective endocarditis is a rare and poorly understood complication of fungemia. Given the rarity of this infection, few studies are available on Fungal IE. Most epidemiologic data are derived from case reports. This study was conducted to explore the clinical characteristics, treatment patterns, and outcomes of patients with Fungal IE.

Methods We conducted a prospective, observational study in the Fuwai Hospital, National Center for Cardiovascular disease, including all consecutive patients with a definite diagnosis of IE admitted from January 2006 through December 2011. The overall characteristics and risk factors for death from FE were analysed.

Results Between January 2006 and December 2011, a total of 22 patients with fungal infective endocarditis were identified. The mean age at presentation was $32 \pm 6$ years, with a slight male preponderance. There were $16(78 \%)$ patients with involvement of a mechanical prosthesis; the majority (64\%) had early prosthetic valve endocarditis (PVE), five cases (23\%) of native valve endocarditis (NVE), one case of pacemaker endocarditis. None of them were intravenous drug users. Most patients (82\%) had healthcare-associated IE. The aortic valve was most commonly affected, and the most common aetiological agent was Candida species, followed by Histoplasma capsulatum, filamentous fungi and pharyngeal aspergillus. The most common symptom at presentation was persistent infection(77\%), followed by weight loss, major vessel embolism, and Anaemia. Major complications occurring during the acute infective phase were also recorded, including renal infarction (73\%), New York Heart Association class III-IV heart failure (45\%), and neurological complication (36\%). Initial therapy consisted of a combination of antifungals in 12 of 22 patients (55\%). Eight patients $(36 \%)$ underwent valve replacement. Pathological evaluation of valve material was of high yield, with organisms identified in $87 \%$ of cases who underwent valve replacement surgery. Prosthetic valve fungal endocarditis was associated with a high morbidity and mortality, with $77 \%$ of patients experiencing complications and $45 \%$ of patients dying of infection-related disease. The overall hospital mortality rate was $66.7 \%$. A better outcome was observed in patients treated with a combined medical and surgical therapy.

Conclusions Fungal infective endocarditis is an increasingly prevalent and devastating disease in today's highly advancing medical practice. An aggressive approach should be considered in patients with prosthetic intravascular devices and significant risk factors for nosocomial fungemia. Microorganism should be investigated particularly by molecular methods on surgical specimens 